\title{
Comentarios críticos de la Norma UNE 7245 sobre "determinación de los compuestos de azufre contenidos en los áridos"
}

\author{
Prof. Dr. JOSE CALLEJA - IETCC/CSIC - MADRID - España
}

\section{$R E S U M E N$}

Se ponen de relieve en este trabajo ciertas definiciones de la Norma UNE 7245 y las correspondientes de la Instrucción EH-80 que la adopta, en cuanto que la citada Norma puede dar valores erróneos por defecto en la determinación de sulfuros $y$, en general, de compuestos de azufre en áridos para hormigón.

Se llama la atención sobre la conveniencia de revisar el limite de 1,2 por ciento para el contenido máximo tolerable de dichos compuestos, particularmente si están constituidos en exclusiva por piritas.

\section{$S U M M A R Y$}

This paper shows some deficiences of the Spanish Standard Method UNE 7245 and the corresponding deficiences of the Spanish Concrete Code EH-80 adopting it. According to the actual Method, erroneous low values may result in determining sulphides and in general sulphur compounds in cocrete aggregates.

Attention is drawn to the convenience of revisnig the limit of 1.2 per cent for the admisible maximun value of such compounds, particularly if they are exmsively formed bu pyrites.

\section{INTRODUCCION GENERAL}

Es bien sabido que los áridos para hormigón a veces contienen compuestos que les hacen inestables dentro del propio hormigón, pudiendo llegar a ocasionar, con sus alteraciones, el deterioro o destrucción del material.

La alteración puede provenir también de un una reacción superficial o masiva entre el árido y la matriz de pasta de cemento que le rodea. Tal es el caso de los áridos reactivos con los álcalis del cemento o, por mejor decir, con los álcalis totales contenidos en el hormigón o llegados a él, cualquiera que sea su procedencia.

La alteración puede provenir también de un proceso de oxidación de determinados compuestos oxidables contenidos en la masa de los áridos, presentes en la superficie o en las fracturas de los mismos y observables a veces a simple vista.

Entre los compuestos oxidables están los sulfuros metálicos, de hierro divalente - del tipo de la pirita amarilla, de la pirrotina o pirita roja magnética y de la marcasita o pirita blanca rómbica-, así como también de otros metales —piritas ferrosas más complejas como las argentíferas, cobrizas (calcopiritas), arsenicales, de níquel, estaño, manganeso cobalto; o también blendas — sulfuros de zinc (wurtzita), de manganeso, de cadmio, etc.-, galena (sulfuro de plomo), etc.

Cuando se encuentran en los áridos, estos sulfuros se hallan, en general, de forma muy dispersa e irregular, ofreciendo un reparto totalmente heterogéneo en la masa del material. También es variable y no uniforme el tamaño en que se presentan los agregados piritosos, lo cual puede influir en la rapidez y magnitud de sus potenciales acciones noci- 
civas para el hormigón: los efectos no son los mismos si provienen de muchos agregados muy finos y muy diluidos en la masa del material, que si son causados por pocos agregados más voluminosos y concentrados en determinados puntos del mismo. La acción de los primeros puede ser extensa pero poco intensa, mientras que la de los segundos puede ser localizada pero mucho más intensa. El resultado final dependerá de las circunstancias particulares de cada caso.

Por todo ello parece tener poco sentido la determinación y hasta la limitación de los sulfuros, particularmente piritosos, en áridos para hormigón, así como su expresión en tanto por ciento de dichos materiales, pues la representatividad de los resultados y su significado dependerán del tamaño de la muestra tomada — que, por lo dicho, indudablemente tendrá que ser grande y, en todo caso, mayor que lo usual一, de la forma de tomarla y tratarla, del grado de finura al que se reduzca... y, en buena medida, del azar. En tales condiciones, ni las muestras ni los resultados que de ellas se obtengan pueden ser, en general, suficientemente representativos.

En el caso de la oxidación de las piritas las presiones internas, a veces localizadas e intensas, se deben a los productos de la oxidación, los cuales se forman por diversos mecanismos químicos, y a su vez pueden desencadenar otros procesos secundarios, igualmente expansivos, cuyos efectos, más o menos diferidos, se suman a los de las acciones primarias.

Entre éstas figuran la formación de oxi-hidróxidos de hierro más o menos hidratados, de ácido sulfúrico y de sulfatos; y entre los procesos secundarios, la formación de ettringita y/o de yeso cristalizado, ambos con carácter expansivo, siempre potencial y a veces real.

En una palabra, los sulfuros pueden actuar sobre el hormigón degradándolo por fisuración producida por dos mecanismos atrbuibles a procesos de oxidación, tanto del anión como de los cationes.

La oxidación del anión sulfuro conduce a la formación del anión sulfato, esquemáticamente representada así:

$$
\mathrm{S}^{2-} \stackrel{2 \mathrm{O}_{2}}{\longrightarrow} \mathrm{SO}_{4}^{2-}
$$

la cual, a su vez, puede dar lugar a yeso en primera instancia:

$$
\mathrm{SO}_{4}{ }^{2-}+\mathrm{Ca}^{2+} \stackrel{2 \mathrm{H}_{2} \mathrm{O}}{\longrightarrow} \mathrm{CaSO}_{4} \cdot 2 \mathrm{H}_{2} \mathrm{O}\left(\mathrm{CS}_{\overline{\mathrm{S}}}{ }_{2}\right)
$$

y a ettringita en segunda:

$$
\begin{array}{ll}
3 \mathrm{C} \overline{\mathrm{SH}}_{2}+\mathrm{C}_{3} \mathrm{AH}_{6} \stackrel{19 \mathrm{H}}{\longrightarrow} \mathrm{C}_{3} \mathrm{~A}(\mathrm{C} \overline{\mathrm{S}})_{3} \mathrm{H}_{31} \\
3 \mathrm{C} \overline{\mathrm{SH}}_{2}+\mathrm{C}_{4} \mathrm{AH}_{13}{ }_{19} \stackrel{13(7) \mathrm{H}}{\longrightarrow} \mathrm{C}_{3} \mathrm{~A}(\mathrm{C} \overline{\mathrm{S}})_{3} \mathrm{H}_{31}+\mathrm{CH}
\end{array}
$$

lo cual puede producir microfisuras por expansión en la pasta cementante.

La oxidación simultánea de los cationes da lugar a la formación de oxi-hidróxidos de hierro hidratados, más o menos complejos, representada en una de las múltiples posibles formas esquemáticas, así: 


$$
\begin{aligned}
& 2 \mathrm{Fe}^{2+}+4 \mathrm{OH}^{-} \\
& 2 \mathrm{Fe}(\mathrm{OH})_{2}+ 1 / 2 \mathrm{O}_{2}+\mathrm{H}_{2} \mathrm{O} \stackrel{n \mathrm{H}_{2} \mathrm{O}}{\longrightarrow} 2 \mathrm{Fe}(\mathrm{OH})_{2} \\
&\left.2 \mathrm{Fe}^{2+}+4 \mathrm{OH}^{-} \frac{\mathrm{O}+\mathrm{H}_{2} \mathrm{O}}{n \mathrm{H}_{2} \mathrm{O}} \rightarrow 2 \mathrm{Fe}(\mathrm{OH})_{3} \cdot n \mathrm{H}_{2} \mathrm{O}\right)_{3} \cdot n \mathrm{H}_{2} \mathrm{O}
\end{aligned}
$$

que por aumento de volumen in situ (topoquímico) pueden producir macrofisuras con carácter disruptivo local en la masa total del hormigón.

En resumen, los sulfuros en general, y las piritas en particular, contenidos en los áridos, son potencialmente nocivos para el hormigón, en virtud de su acción a través de los complejos mecanismos de oxidación e hidratación, y de formación y cristalización expansiva de compuestos (1) (2) (3) señalados esquemáticamente en lo que precede. Por esta razón los áridos que contienen piritas aún en pequeñas cantidades, son considerados como inestables y peligrosos (3) (4), por lo que se ha entendido que su utilización debe ser proscrita (5).

\section{ANTECEDENTES}

En España se han detectado y denunciado casos en los que los deterioros de elementos y estructuras de hormigón producidos por alteración de áridos piritosos han sido numerosos e importantes, estando provocados por un complejo conjunto de acciones destructivas del hormigón y de la adherencia entre los áridos gruesos y la pasta o el mortero cementante, o entre éstos y las armaduras, así como por la corrosión secundaria de éstas y por la propia expansión de los productos formados en el proceso corrosivo.

En este sentido se han puesto de manifiesto, tiempo ha, casos atribuidos a áridos procedentes de la cantera de Mont-Palau, de Pineda de Mar, y que han afectado a las zonas de El Maresme y La Selva, así como a Barcelona y el Prat de Llobregat, en Cataluña (6) (7) (8) (9).

En estos casos se han podido observar fisuraciones y agrietamientos en prefabricados de forjados de piso, viguetas pretensadas, forjados semirresistentes, hormigón armado de pilares y hormigón de relleno (7).

\section{COMENTARIOS CRITICOS DE LA NORMA UNE 7245}

Como consecuencia de las casos mencionados, la Norma UNE 7245 para la "Determinación de los compuestos de azufre contenidos en los áridos", ha sido puesta en entredicho, y aun impugnada, en el sentido de que las cantidades de estos compuestos -expresadas en tanto por ciento en peso de $\mathrm{SO}_{4}{ }^{2-}$, referido al árido seco-, encontradas en los áridos analizados, son - han sido- mucho menores que las realmente existentes en los mismos (6) (9).

Esto no es de extrañar, dado el método descrito en la citada Norma. En efecto, ésta señala literalmente como objeto de la misma el conocimiento cualitativo de "la existencia en los áridos de compuestos de azufre solubles en agua o en ácido clorhídrico y los sulfuros oxidables durante la hidratación y, en su caso, apreciarlo cuantitativamente".

Aparte de la redación defectuosa del entrecomillado anterior, su contenido no concuerda con el de los puntos siguientes de la Norma, relativos a los ensayos cualitativos y a la determinación -que no "investigación"- cuantitativa de sulfuros. En efecto, centran- 
do el tema en los sulfuros, punto más débil del método, cabe hacer las siguientes consideraciones.

El método se refiere a los compuestos de azufre oxidables "durante la hidratación", sin precisar cuáles ni en qué condiciones. $\mathrm{Y}$ hay que suponer que, no sólo oxidables durante la hidratación, sino también, y lo que es más importante y peligroso, después de la hidratación, es decir, cuando el material ya está fraguado y endurecido, lo cual es asimismo posible y mucho más nocivo para el hormigón.

Hay que suponer también al respecto, que no se trata de sulfuros de metales ligeros -alcalinos y/o alcalinotérreos-, los cuales se descompondrían por hidrólisis al ponerse los áridos que los contienen, en contacto con el agua de amasado; aparte de que no sería probable su presencia en áridos precisamente, al contrario de lo que podría ocurrir en el caso de algunas escorias siderúrgicas y de los correspondientes cementos que las contuviesen —-sulfuro cálcico-.

Por el contrario, cabe suponer que se trata de sulfuros de metales pesados — principalmente de hierro (ferroso)-, los cuales pueden resistir mejor, sin descomponerse, la acción hidrolítica del agua y el ataque por algunos ácidos en determinadas condiciones de concentración y fuerza de éstos, y de tiempo y temperatura de ataque por parte de los mismos.

En el caso de tales sulfuros, y de acuerdo con el modus operandi de la Norma UNE 7245, cabe hacer dos consideraciones: una respecto del ataque ácido de la muestra de árido para determinar en ella - cualitativa o cuantitativamente- los sulfatos solubles; y otra, relativa a la oxidación a sulfatos de posibles sulfuros existentes en dicha muestra.

En cuanto a la primera consideración, hay que pensar que, si el ataque ácido es suficientemente fuerte y prolongado, parte del (o de los) sulfuro(s) del árido se podría descomponer, con desprendimiento de gas sulfhídrico $\mathrm{SH}_{2}$, que al escapar sustraería a la determinación cuantitativa de sulfuros en forma de sulfatos, la parte de azufre correspondiente. Algo parecido sucedería con los sulfitos, bisulfitos, tiosulfatos, politionatos, etc., los cuales se descompondrían con cesión de gas sufuroso $\mathrm{SO}_{2}$.

Así, pues, los métodos para la determinación analítica de sulfuros, basados en ataques oxidantes con ácidos de una cierta fuerza como tales, son inseguros (8), por cuanto que parte del azufre de valencias inferiores a seis (S VI) que se encuentre en los áridos o en el hormigón formando compuestos de azufre distintos de los sulfatos $\multimap$ por ejemplo, sulfuros (S II) o sulfitos (S IV), etc.-, puede desprenderse en forma de gases sulfhídrico o sulfuroso, escapando a la determinación. En consecuencia, ésta puede dar resultados erróneos por defecto (8) (10).

En cuanto a la segunda consideración, hay que tener en cuenta que si el tratamiento oxidante no es lo suficientemente intenso y prolongado, una parte de los sulfuros - "restantes" del ataque ácido previo- puede no llegar a oxidarse, con lo oual se determinarían menos de los que correspondería determinar, obteniéndose asimismo resultados erróneos por defecto.

El ácido clorhídrico 1:3 utilizado según el método normalizado UNE 7245 para solubilizar los sulfatos, parece suficientemente fuerte como para llevar a cabo la solubilización de los mismos en las condiciones señaladas de tiempo de ebullición, y no lo suficientemente fuerte como para descomponer - al menos totalmente - las piritas que se pudieran encontrar en el árido analizado. Sin embargo, es un hecho que los áridos pueden contener sulfuros atacables por ácido clorhídrico de dicha concentración y en caliente, y que algu- 
nas piritas detectadas a simple vista en la superficie de los áridos y confirmadas como tales por DRX se atacan con ácido clorhídrico de dicha concentración, con desprendimiento de gas sulfhídrico de olor característico, como se ha podido comprobar. Es evidente que estos sulfuros y estas piritas no podrían ser determinados cuantitativamente por el método de la Norma UNE 7245, aun cuando fueran oxidables cuantitativamente por ácido nítrico sin más. Y lo mismo sucede, aún con mayor razón que en el caso de las piritas, con las combinaciones oxigenadas de azufre de grado de valencia inferior a seis, también tenidas en cuenta implícitamente en la Norma UNE 7245.

El ácido nítrico 1:1 ("diluido", según la Norma UNE 7245) es previsible que, en frío opere como ácido en lugar de $\multimap$ además de- como oxidante, con lo cual actúa sobre los sulfuros atacables por ácidos y sobre las combinaciones oxigenadas de S VI, y tal vez parcialmente también sobre ciertas piritas, lo mismo que el ácido clorhídrico, agravando la situación. Pero, además es posible que ni en frío ni en caliente tenga el potencial oxidante necesario para oxidar cuantitativamente a sulfatos, a todos los compuestos de azufre "restantes" de "los" ataques ácidos previos (clorhídrico y nítrico).

Las consideraciones precedentes llevan a la conclusión de que si se dan aisladamente e indistintamente cualquiera de las circunstancias anteriores, o si se dan ambas a la vez, la determinación de sulfuros en forma de sulfatos puede ser notablemente errónea por defecto, y más, como es natural, en el caso de simultaneidad de ambas circunstancias.

Por ello precisamente los métodos analíticos más acreditados desde antiguo para la determinación de sulfuros en forma de sulfatos y en general para la oxidación de todo azufre en grado de valencia inferior a seis, suelen operar en medios con un potencial oxidante muy fuerte. Así, por ejemplo, en el caso de las piritas en general se opera con agua regia $\mathrm{HCl} / \mathrm{HNO}_{3}=3: 1$, con ácido clorhídrico y clorato potásico o agua regia y clorato potásico (11), con ácido nítrico fumante, o mejor con bromo y ácido nítrico. $O$, lo que es todavía mucho mejor para evitar el medio ácido, por fusión alcalina oxidante con peróxido de sodio, o con una mezcla de carbonato sódico y nitrato potásico 10:1 (12), o por disgregación con hidróxido y peróxido de sodio en una primera etapa y tratamiento con bromo en una segunda, de acuerdo con la descripción detallada del método que preconiza este procedimiento (10).

En todo caso debe quedar bien claro que por el método descrito en la Norma UNE 7245 teóricamente se determina en forma de sulfato el total de compuestos de azufre con cualesquiera grados de valencia, por una parte; y complementariamente los sulfatos (S VI) como tales, por otra. Con lo cual todos los compuestos de azufre que no están en forma de sulfatos - y no sólo los sulfuros (S II) - se calculan por diferencia y se atribuyen en su totalidad, en algunos casos indebidamente, a sulfuros. Aparte de ello están las causas de error por defecto antes indicadas.

No obstante, dado el peligro potencial que los áridos con sulfuros pueden representar para la estabilidad del hormigón, la determinación para el conocimiento de su presencia y de su contenido en aquéllos y en éste no se puede soslayar, pese a todos los inconvenientes, dificultades e incertidumbres señalados. Como tampoco se puede eludir el interés o la necesidad de fijar un límite racional para dicho contenido, con vistas a garantizar la mencionada estabilidad del hormigón. En este sentido hay descritos métodos más idóneos que permiten determinar en exclusiva los compuestos de azufre en estado de sulfuro, bien basados en su oxidación (10), o bien basados en el desprendimiento de gas sulfhídrico por ataque ácido (11), tal y como se describe para los cementos (13). Aplicados estos últimos métodos a los áridos se deberá comprobar que no tiene lugar el proceso

$$
\mathrm{SO}_{2}+\mathrm{SH}_{2} \rightarrow 2 \mathrm{~S}+\mathrm{H}_{2} \mathrm{O}
$$


en su caso -es decir, en presencia de S IV - , lo cual obligaría a reoxidar con agua regia y clorato potásico el azufre formado, complicándose mucho la determinación. En consonancia con lo indicado anteriormente, ni tođo el azufre oxidado sería atribuible en exclusiva a S II, ni tampoco a S IV, en un caso tall (11).

Una última observación cuando se aplican los métodos oxidantes al hormigón y a sus áridos es que, por tratarse, en general, de materiales áltamente silícicos, es preciso proceder a la separacón de la sílice, previamente a la precipitación del S VI como sulfato bárico en medio clorhídrico, por la perturbación que dicha sílice puede ejercer en la determinación de los sulfatos (10).

\section{COMENTARIOS CRITICOS ACERCA DE LA INSTRUCCION EH-80}

La primera objeción que se puede hacer de la Instrucción EH-80 y de su predecesora la EH-73 (14) es la de apoyarse en la Norma UNE 7245, en cuanto al método para determinar sulfuros oxidables, aparte de la observación aclaratoria, que se hace aquí una vez más, de que dicha Norma considera implícitamente, pero no explícitamente, la posibilidad de existencia de otros compuestos oxidables de azufre, distintos de los sulfuros $-\mathrm{y}$ además de los sulfatos-.

Se ha suscitado la duda de si la limitación máxima de $1,2 \%$ para el contenido de compuestos de azufre expresados como $\mathrm{SO}_{4}{ }^{2-}$ en los áridos, determinado por el método de la Norma UNE 7245, garantiza la estabilidad de los mismos cuando se refiere exclusivamente a azufre de piritas o si, por el contrario, en tal caso dicha limitación podría considerarse alta (8).

A este respecto cabría decir que sería muy difícil establecer una limitación máxima, incluso referida exclusivamente a sulfuros metálicos pesados — piritas-, tal que proporcionase una garantía semejante. Fuera la que fuere tal limitación, sería previsible que áridos con contenidos superiores al límite fijado fuesen más estables que otros con contenidos inferiores al mismo, y viceversa.

Ello sería debido al hecho, ya señalado, de que la estabilidad o inestabilidad mayor o menor no dependería, ni en exclusiva ni tal vez en primer lugar, de la cantidad absoluta de pirita en el árido, sino más bien del tamaño de los núcleos piritosos y de su reparto más o menos regular y homogéneo, y de su proporción en la superficie del mismo; también de su estado y naturaleza - cristalinidad- del sulfuro metálico ferroso, por cuanto que ello puede influir en su mayor o menor facilidad y velocidad de oxidación y en el grado alcanzado por la misma, tal y como se indicaba en la Introducción General. Y, por supuesto, también dependería de la calidad del hormigón y de las circunstancias externas del mismo (entorno, ambiente y condiciones de servicio), entre otras muchas cosas.

Si se tienen en cuenta todos los estados posibles del azufre en los áridos $-\mathrm{y}$ lo mismo en el hormigón-, tal vez el valor de $1,20 \%$ pudiera ser aceptable como límite superior para el conjunto de todos los dichos estados. Pero tal vez no lo fuera siempre, dependiendo del "reparto" de dicho valor entre los posibles de tales compuestos presentes en cada caso en los áridos.

Por ejemplo, no sería lo mismo que ese $1,20 \%$ estuviera constituido exclusivamente -o casi- por sulfatos (como suele ser el caso general), o que, por el contrario, estuviera muy mayoritariamente constituido por sulfuros - piritas-. Este segundo caso podría ser más grave para el hormigón y, en tal sentido, dicho valor limitativo podría ser incluso alto en demasía. 
De cualquier modo, parecería conveniente estudiar de forma experimental y variada una limitación superior exclusiva de sulfuros oxidables en los áridos y en el hormigón, así como establecer un método fiable para su determinación. Tanto para lo uno como para lo otro parece haber sugerencias válidas (10) que valdría la pena estudiar más a fondo.

\section{CONCLUSIONES}

Como conclusiones de lo que precede pueden establecerse las siguientes:

5.1. Los áridos para hormigón que contienen compuestos de azufre "inorgánico" en cualquier grado de valencia y en unas determinadas proporciones, pueden ser potencialmente expansivos por diversos mecanismos y con variados efectos destructivos para el material. En particular lo son los que contienen sulfuros de hierro en sus diversas formas.

5.2. La Norma UNE 7245 para la determinación de los compuestos de azufre en los áridos para hormigón no es idónea para dicho fin, por cuanto que puede dar lugar en muchos casos a resultados erróneos por defecto y señalar contenidos de pirita en los áridos, inferiores a los reales.

5.3. La determinación de sulfuros en los áridos para hormigón debería hacerse por métodos que permitieran llevarla a cabo de manera independiente y en exclusiva.

5.4. La Instrucción EH-80 y cuantas hayan adoptado la Norma UNE 7245 deberían tener en cuenta las conclusiones anteriores 5.2. y 5.3.

5.5. Convendría que el valor de $1,20 \%$, máximo tolerado para el contenido de compuestos de azufre en áridos tanto gruesos como finos para hormigón, fuese revisado en el caso de referirse exclusivamente a piritas.

\section{RECOMENDACIONES}

En virtud de las conclusiones anteriores se sugiere tener en cuenta las siguientes:

6.1. Modificar o sustituir la actual Norma UNE 7245 por otra que carezca de sus defectos.

6.2. En su consecuencia, estudiar y proponer un método analítico que permita la determinación directa y exclusiva de los sulfuros en los áridos, eligiendo tal vez entre los métodos ya existentes y descritos (10).

6.3. Llevar a cabo una experimentación para determinar si la limitación de 1,2 \% para los compuestos de azufre en los áridos sigue siendo válida cuando dichos compuestos corresponden a pirita en exclusiva.

6.4. En consecuencia con las recomendaciones anteriores, modificar adecuadamente en su momento la Instrucción EH-80 y todas las que tengan adoptada la Norma UNE 7245 y fijado el valor de $1,2 \%$ como máximo tolerable de compuestos de azufre en áridos para hormigón. 
(1) Symposium on Mineral Aggregates, STP No. 83, pág. 39 ASTM 1916 Race St. Philadelphia 3, Passadena, U.S.A., 1948.

(2) Journal of the American Concrete Institute, pág. 1.000, Mayo 1956.

(3) Norma DIN 4226: "Disposiciones del Comité Alemán para Hormigón Armado: Aridos para Hormigón procedentes de Yacimientos Naturales", 1955.

(4) A. KLEINLOGEL: "Influencias fisicoquímicas sobre hormigones en masa y armados". Editorial LABOR, S. A., págs. 247, 258, Madrid-Barcelona, 1955.

(5) O. Valenta: V Congreso Internacional de Química del Cemento, Parte III, pág. 202, Tokyo, 1968.

(6) E. MACH y L. Roca: "Muchos edificios en peligro a causa de ciertos áridos". Primeras Jornadas de Durabilidad del Hormigón. Materiales de Construcción (IETCC), núm. 150-151, 107-120 (1973).

(7) J. OVEJERo: “¿Elementos estructurales de hormigón condenados a una ruina total sin remedio?”. Primeras Jornadas de Durabilidad del Hormigón. Materiales de Construcción (IETCC), núm. 150-151, 121 (1973).

(8) N. MaJo: "Reflexiones sobre el caso de las piritas de El Maresme". Primeras Jornadas de Durabilidad del Hormigón. Materiales de Construcción (IETCC), núm. 150-151, 123-144 (1973).

(9) J. Calleja: "Las Primeras Jornadas de Durabilidad del Hormigón IETCC (Madrid, Octubre 1972)". Cemento-Hormigón (467), 351-353 y 354-356 (1973).

(10) F. Goma: "Método utilizado para la determinación analítica de sulfuros y de azufre total contenidos en áridos y hormigones, y consideraciones acerca de la necesidad de separar, del azufre total tolerable en los áridos, el debido a la presencia de sulfuros". Materiales de Construcción (IETCC), número $156,51-55$ (1974).

(11) F. P. Treadwell y W. D. Treadwell: "Tratado de Química Analítica". Manuel Marín y Cía., Editores. Barcelona-México, 1958, págs. 317-323.

(12) I. M. KolthofF y E. D. SANdelL: "Textbook on Quantitative Analysis". The Macmillan Company, New York, 1948, págs. 342-343.

(13) RC-75: Pliego de Prescripciones Técnicas Generales para la Recepción de Cementos. MOPU, Madrid, 1975.

(14) EH-80: Instrucción para el Proyecto y la Ejecución de Obras de Hormigón en Masa y Armado. MOPU, Madrid, 1980, y su precedente: FH-73, MOPU, Madrid, 1973.

\section{publicación del i.e.t.c.c.}

El Instituto Eduardo Torroja, miembro activo tanto del Comité Eurointernacional del Hormigón (CEB), como de la Federación Internacional del Pretensado (FIP), ha tomado a su cargo la traducción y edición de esta imporante normativa.

Aunque presentado con el titulo de "Código Modelo CEB i FIP 1978" este documento incorpora los dos primeros volümenes de este "Sistema Unificado Internacional de Reglamentación Técnica de Ingenieria Civil». El primer volumen de este "Sistema Unificado" es el denominado "Reglas comunes Unificadas para los diferentes tipos de obras y materiales", donde se exponen los criterios y formatos de seguridad a que han de ajustarse los diferentes Códigos (estructuras de hormigón, estructuras metálicas, estructuras mixtas, estructuras de albañileria y estructuras de madera), que han de configurar la totalidad del antedicho sistema.

El segundo volumen es propiamente el Código Modelo para las Estructuras de Hormigón. Fruto de la colaboración de dos asociaciones del prestigio del CEB y la FIP, desde mediados de los 60, incorpora los avances cientificos $y$ tecnologicos producidos en los uiltimos años sin detrimento alguno de la claridad y operatividad que deben presidir un código que pretende ser, ante todo, un auxiliar práctico para los técnicos de la construcción.

El Código sigue en su estructura las reglas más o menos clásicas: una primera parte dedicada a los datos generales para el cálculo (propiedades de los materiales, datos relativos al pretensado, tolerancias); en segundo lugar se presentan las reglas de proyecto estructural lacciones, solicitaciones, estados limites ultimos y de utilización, reglas de detalle para el armado); y, por último, ejecución, mantenimiento y control de calidad.

Tambièn incluye reglas para estructuras con elementos prefabricados y estructuras de hormigón con áridos ligeros. Los Anejos del Código se refieren a: terminologia, proyecto mediante la experimentación, resistencia al fuego código-modelo ceb-fip tecnologia del hormigón, comportamiento en el tiempo del hormigón y fatiga. 